\title{
Fizeau's "aether-drag" experiment in the undergraduate laboratory
}

\section{Renaud Mathevet, Pierre Labastie, Thierry Lahaye}

Renaud Mathevet, Pierre Labastie, Thierry Lahaye, "Fizeau's "aether-drag " experiment in the undergraduate laboratory," Proc. SPIE 9793, Education and Training in Optics and Photonics: ETOP 2015, 97931G (8 October 2015); doi: $10.1117 / 12.2223112$

SPIE Event: Education and Training in Optics and Photonics: ETOP 2015, 2015, Bordeaux, France 


\title{
Fizeau's "aether-drag" experiment in the undergraduate laboratory
}

\author{
Renaud Mathevet ${ }^{a^{*}}$, Pierre Labastie ${ }^{b}$ and Thierry Lahaye ${ }^{c}$ \\ ${ }^{a}$ LNCMI, UPR 3228, CNRS-INSA-UJF-UPS, Grenoble and Toulouse, France ; \\ ${ }^{b}$ LCAR, IRSAMC, UMR 5589, CNRS-Université de Toulouse, Toulouse, France ; \\ ${ }^{c}$ LCF, UMR 8501, CNRS-Institut d'Optique-Université Paris Sud 11, Palaiseau, France ;
}

\begin{abstract}
We describe a simple realization of Fizeau's "aether-drag" experiment. Using an inexpensive setup, we measure the phase shift induced by moving water in a laser interferometer and find good agreement with the relativistic composition of velocities law or, in the terms of 19th century physics, with Fresnel's partial-drag theory.

This appealing experiment, particularly suited for an undergraduate laboratory project, not only allows a quantitative measurement of a relativistic effect on a macroscopic system but also constitutes a practical application of important concepts of optics, data acquisition and processing, and fluid mechanics.
\end{abstract}

Keywords: special relativity, interferometry

\section{INTRODUCTION}

In introductory courses and textbooks dealing with special relativity, Fizeau's "aether-drag" experiment often appears simply as an application of the relativistic law of composition of velocities. However, Albert Einstein himself declared that Fizeau's measurement of the speed of light in moving water was, together with stellar aberration, one of the experimental results that had influenced him most in the development of relativity [1]. In spite of this high praise, introductory expositions of Fizeau's experiment, including a discussion of its historical development and the details of the experimental setup, are often lacking. Moreover, many textbooks actually show incorrect experimental arrangements that would not allow for the observation of the effect in practice. Here, we show that one can actually perform Fizeau's experiment with rather modest equipment, and that such a project illustrates in an appealing way not only relativistic kinematics but also interesting aspects of wave optics, data acquisition and processing, and even fluid mechanics.

\section{THEORETICAL BACKGROUND}

In this section, we recall the derivation of the phase difference $\Delta \varphi$ induced by the motion, with velocity $\mathrm{v}$, of the medium of refractive index $\mathrm{n}$ in the interferometric arrangement shown in Fig. 1, which is essentially the one used by Michelson and Morley in 1886 [2].

Let us consider first the case where water and monochromatic light of vacuum wavelength $\lambda$ propagate in the same direction. In the reference frame where water is at rest, the phase velocity of light is $\mathrm{c} / \mathrm{n}$. In the laboratory frame, using the relativistic composition of velocities [3], the phase velocity of light is

$$
v_{+}=\frac{c / n+v}{1+(c / n) v / c^{2}} \text {. }
$$

The phase accumulated by light over the propagation distance of $2 \mathrm{~L}$ is thus

$$
\varphi_{+}=\frac{2 \pi}{c} \frac{2 L}{v_{+}} .
$$

Where light and water propagate in opposite directions, the corresponding phase is obtained by replacing $\mathrm{v}$ by $-\mathrm{v}$ in Eq. (1). The phase difference between the two arms of the interferometer at first order in v/c reads

*Further author information: renaud.mathevet@lncmi.cnrs.fr 


$$
\Delta \varphi_{\text {rel. }}=2 \pi \frac{v}{c} \frac{4 L}{\lambda}\left(n^{2}-1\right) .
$$

Using the non-relativistic addition of velocities, i.e., by replacing $\mathrm{v}_{ \pm}$by $\mathrm{c} / \mathrm{n} \pm \mathrm{v}$ one gets

$$
\Delta \varphi_{\text {non-rel. }}=2 \pi \frac{v}{c} \frac{4 L}{\lambda} n^{2},
$$

which has the same functional form except for a coefficient $n^{2}$ in place of $n^{2}-1$. In Fresnel's language, this would correspond to a complete aether-drag. The ratio of the predictions (Eqs. (4) and (3)) is about 2.3 for water (n 1.33).

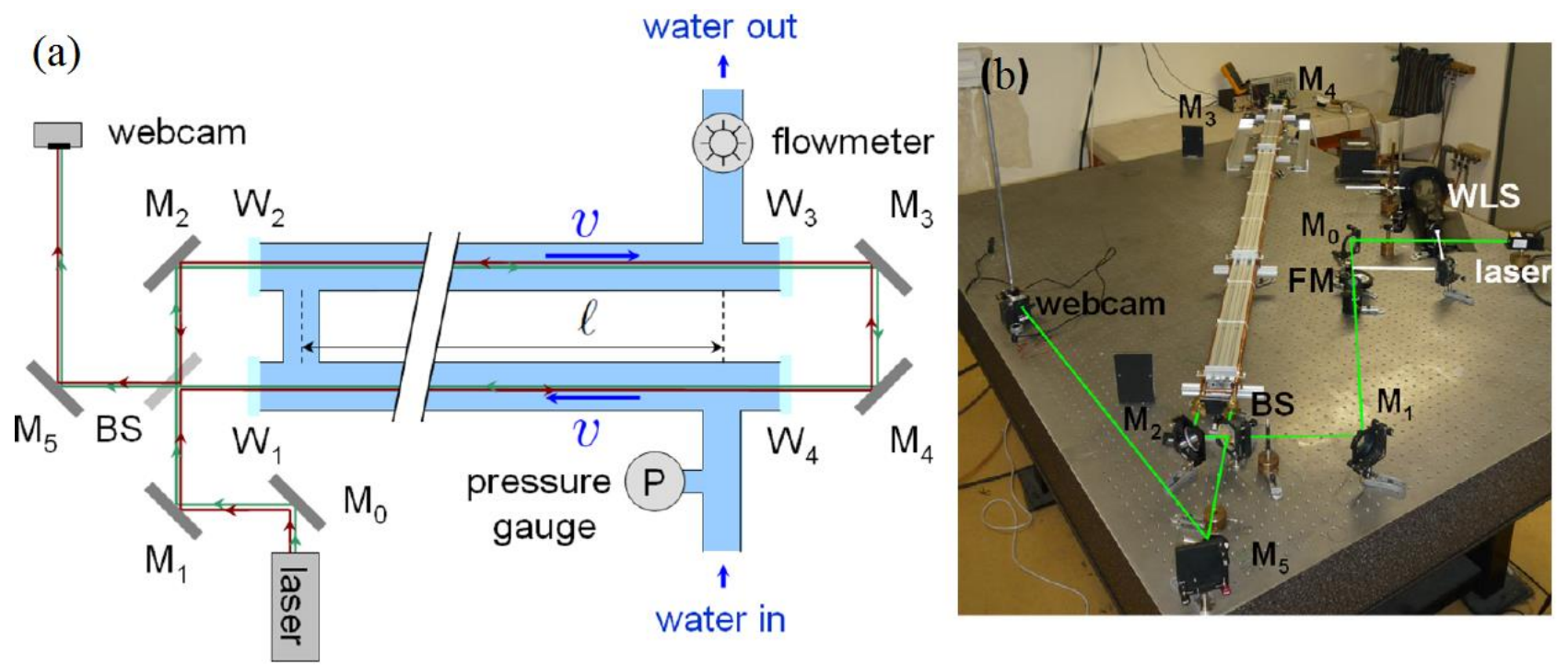

Figure 1: Experimental setup (a) Sketch. Mi: mirror; Wi: windows; BS: beam splitter. The two counter-propagating beams are drawn in red and green for clarity. (b) Picture. WLS: white light source; FM: flipping mirror.

\section{EXPERIMENTAL RESULTS}

For simplicity and to keep costs low, we built our system from standard $8 \mathrm{~mm}$ in diameter copper piping materials available in any hardware store and use water directly from the tap allowing us to reach flow velocities up to $6 \mathrm{~m} / \mathrm{s}$. The water pressure is varied using the tap valve and measured with a pressure gauge connected to the inlet port. We get a continuous measurement of the flow rate $\mathrm{Q}$ using a paddlewheel flowmeter. The two 2-m long copper pipes are relatively flexible and soft. Their straightness and parallelism are ensured by fastening them on a slotted, $30 \times 60 \mathrm{~mm}^{2}$ cross-section aluminum profile by means of cable ties (Fig. 1(b)).

The windows are common 3.3-mm thick, $25-\mathrm{mm}$ diameter uncoated borosilicate glass substrates. They are pressed against a brass female 3/4 in BSP cap at the center of which a 6-mm diameter hole is drilled.

As a light source, we use an inexpensive diode-pumped, solid-state laser delivering a quasi-collimated beam with several milliwatts of light at $\lambda=532 \mathrm{~nm}$ and the fringe pattern is recorded a simple webcam whose objective lens and IR filter have been removed in order to directly expose the CMOS detector chip.

Figure 2a shows typical data from which we measure the phase difference as a function of the measured water velocity $v_{\text {meas. }}$. The origin of phases has been chosen to vanish at zero velocity. As can be seen in Fig. $2 b$, we take five measurements for each velocity to improve the statistics and to get an estimate of the variance. We observe a clear linear dependence and a linear fit (thin solid line) gives a slope of $0.274 \pm 0.003 \mathrm{rad} \mathrm{s} / \mathrm{m}$. The non-relativistic prediction of Eq. (4) (dashed line) has slope $0.563 \mathrm{rad} \mathrm{s} / \mathrm{m}$, which does not match the experimental results at all. The relativistic prediction given by Eq. (3) (thick solid line) has slope $0.248 \mathrm{rad} \mathrm{s} / \mathrm{m}$ and is in much better agreement with the experimental data. However, there appears to be a slight systematic error in the data compared with the prediction. This comes from the fact 
that we measure the mean velocity $\mathrm{v}_{\text {meas }}$ averaged over the velocity profile inside the pipes, whereas the velocity $\mathrm{v}$ appearing in Eq. (3) is the velocity at the position of the beam, i.e., on the axis of the pipes.

A theoretical model for the radial dependence is thus required but in the turbulent regime in which we operate no simple rigorous analytic expression for the velocity profile. A commonly used empirical law [2] gives a correction factor of 1.16 and we must add an extra 3.8\% correction due to dispersion. The relativistic expected slope is then $0.299 \mathrm{rad} \mathrm{s} / \mathrm{m}$ (not shown in Fig. 2(b)). The agreement between the experimental and theoretical values is thus at the level of $8 \%$.
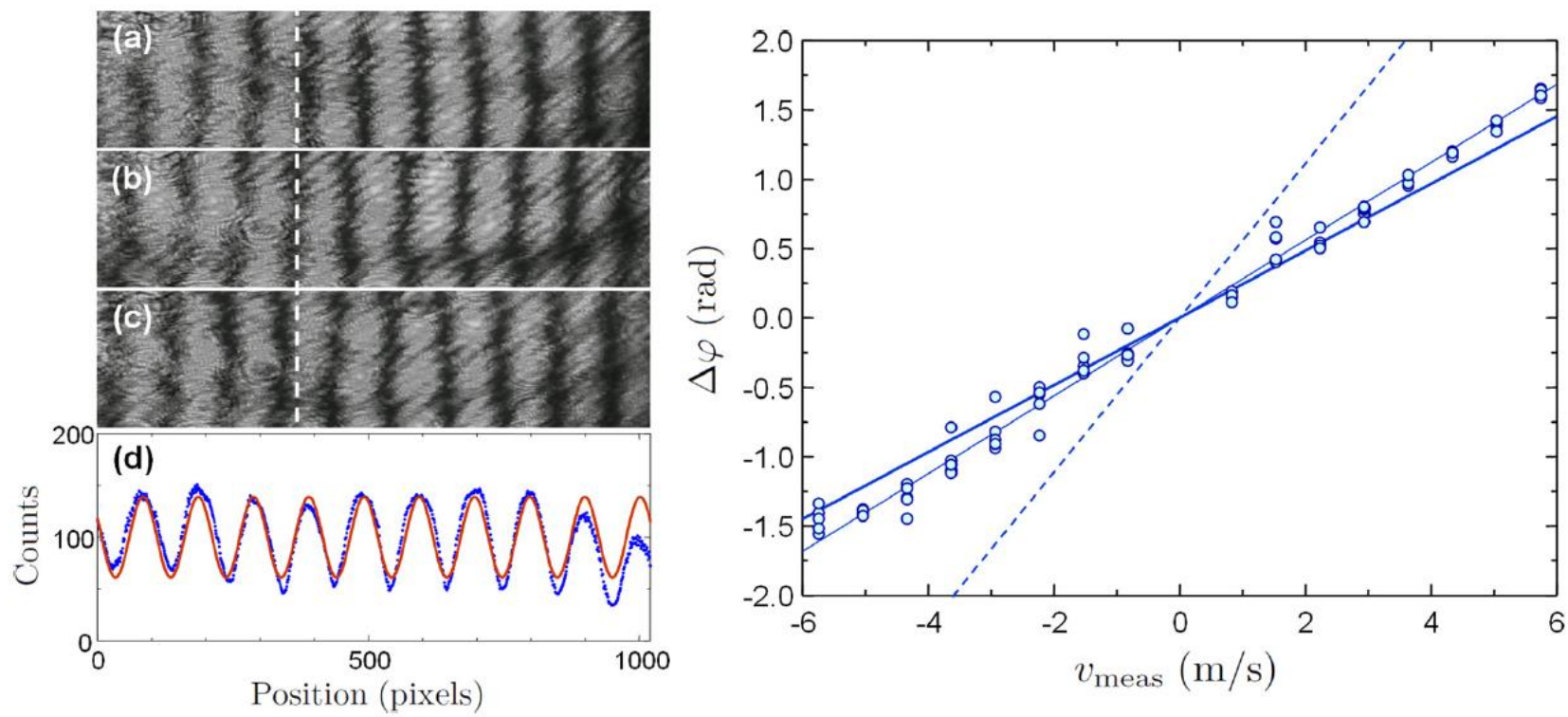

Figure 2: Experimental results. Left: Sample images of the fringe pattern obtained from the camera for (a) $\mathrm{v}=-5.7 \mathrm{~m} / \mathrm{s}$, (b) $v=0.8 \mathrm{~m} / \mathrm{s}$, and (c) $v=5.7 \mathrm{~m} / \mathrm{s}$. The white dashed line shows the position of the central fringe for $\mathrm{v}=0$. (d) Processing of the image shown in (c) - the dots are the vertically integrated intensities and the (red) solid curve is the sinusoidal best fit. Right: measured phase shift as a function of water flow velocity. Circles: data; thin solid line: linear fit, giving a slope of $0.274 \pm 003 \mathrm{rad} \mathrm{s} / \mathrm{m}$; thick solid line: relativistic prediction from Eq. (3); dashed line: non-relativistic prediction from Eq. (4).

\section{DISCUSSION}

Based on our data, we conclude that the non-relativistic prediction is clearly ruled out by our measurements. Nevertheless, the rather good agreement with the relativistic prediction must not be over interpreted. Indeed, it is difficult to put an accurate error bar on the result, as several systematic effects should be studied carefully for such a purpose. First, the systematic errors are dominated by our flowrate measurement. A more careful calibration should thus be performed in order to improve the accuracy. Second, the factor of 1.16 due to the shape of the velocity profile should be measured specifically for our system. A final source of uncertainty is the determination of the actual length $\mathrm{L}$ appearing in Eq. (3-4). In practice, the flow makes a right-angle turn at each end of the pipes, so the velocity will presumably be affected up and downstream on length scales on the order of the pipe diameter $\mathrm{d}$. This implies a correction of order $\mathrm{d} / \mathrm{L}$ (on the percent level), but again, an accurate estimation is difficult.

\section{EXPERIMENT WITH A WHITE LIGHT SOURCE}

We have also performed this experiment using a white light source instead of a laser. The source is a 1-mm diameter iris illuminated by a 55-W halogen lamp (the type used for car headlights) and a condenser lens. The resulting diverging beam is collimated by a 100-mm focal length lens and superimposed onto the path of the laser beam using two mirrors. The second mirror, located between $\mathrm{M}_{0}$ and $\mathrm{M}_{1}$, is a flipping mirror so that one can switch easily between the laser and the white light sources (see Fig. 1(b)).

Once the interferometer has been aligned with the laser, white-light fringes are readily observed. Naturally, if the iris is opened the luminosity is increased at the expense of spatial coherence, and the contrast in the picture is lost. The 
fringes are then localized in the vicinity of mirror M3. A color image as in Fig. 3(a) is then recovered using a converging lens that conjugates M3 and the detector plane.

The advantages of using a white source is that (i) the position of the dark central fringe can be found without ambiguity as the contrast of the colored fringes vanishes rapidly away from the zero path-length difference and (ii) compared to using a laser, unwanted interference fringes (due to scattering on dust particles for example) as well as speckle, are suppressed. There are, however, a certain number of drawbacks. Besides the reduced luminosity, making quantitative comparisons with theory is obviously much more difficult than in the monochromatic case. Indeed, one would need to measure the light spectrum, as well as the wavelength-dependent reflectivity (including phase shifts) introduced by the beamsplitter in order to model quantitatively the fringe pattern. For instance, we have observed that using a metallic beamsplitter instead of the dielectric one significantly alters the colors and the contrast of the fringe pattern obtained in white light. We made a composite of 22 images taken for various water velocities v. The result, shown in Fig. 3(b), clearly shows that the fringe positions shift linearly with velocity. However, a quantitative analysis of such an image is not easy, and the motivation behind this figure is mainly for aesthetics.
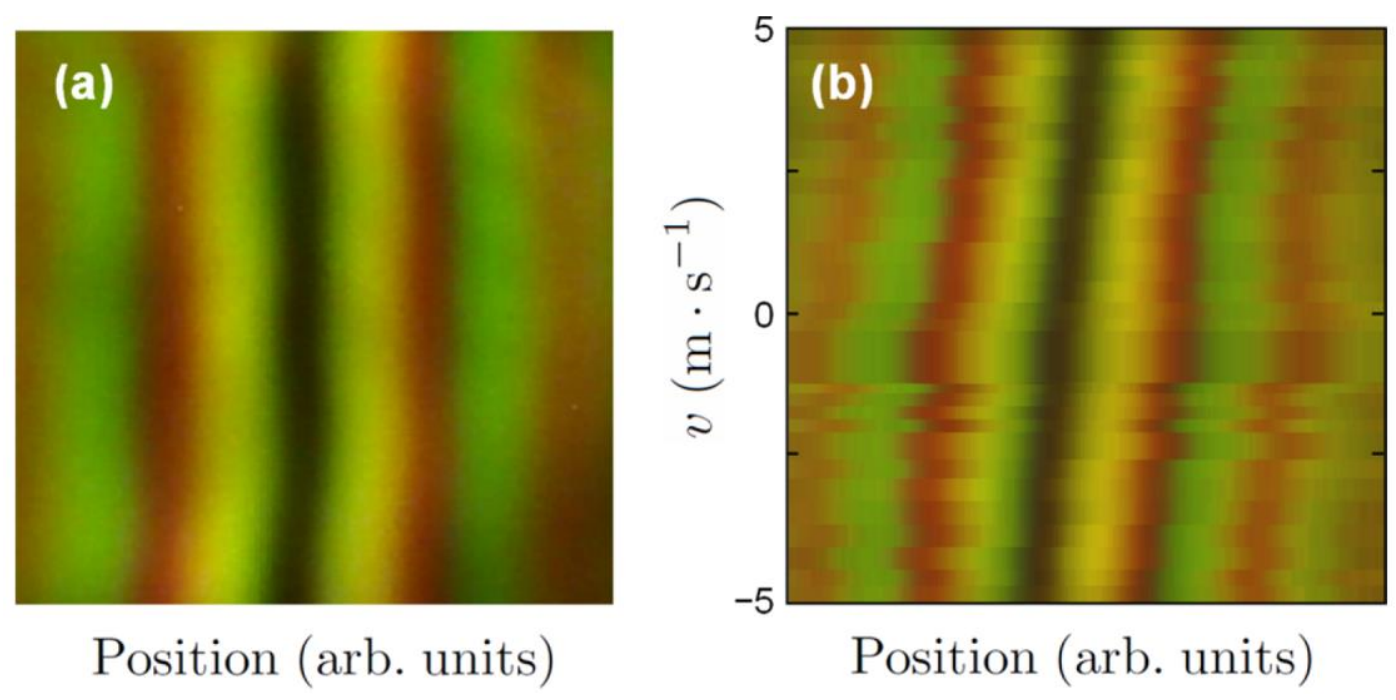

Figure 3: (a) A sample white-light fringe pattern. (b) Composite image of 22 fringe patterns obtained for different water velocities v. One clearly observes the linear shift of the central fringe position as a function of $\mathrm{v}$.

\section{CONCLUSION AND OUTLOOK}

Using rather modest equipment, we have shown that Fizeau's "aether-drag" experiment can be reproduced in the undergraduate laboratory at a quantitative level [4]. It not only makes a nice practical introduction to the sometimes abstract concepts of special relativity but also constitutes an interesting application of several branches of experimental physics. Immediate improvements of the setup described in this paper would consist of (i) calibrating the flowrate more carefully and (ii) increasing the stiffness of the tubing system. A natural extension of this work, suitable for a long-term student project, would consist of trying to study the systematic effects in detail - for example, the determination of the effective length L. One way to measure this effect would be to start from the full pipe length and then repeat the experiment for shorter and shorter pipe lengths. The effect can then be evaluated by measuring the dependence of the slope $\Delta \varphi / v$ on the pipe length.

\section{REFERENCES}

1. R. S. Shankland, “Conversations with Albert Einstein,” Am. J. Phys. 31, pp. $47-57$ (1963).

2. A. A. Michelson and E. W. Morley, "Influence of Motion of the Medium on the Velocity of Light," Am. J. Sci. 31, pp. 377-386 (1886).

3. E. F. Taylor and J. A. Wheeler, Spacetime Physics: Introduction to Special Relativity, 2nd ed. (W. H. Freeman \& Company, New York, 1992).

4. T. Lahaye, P. Labastie and R. Mathevet, "Fizeau's aether-drag experiment in the undergraduate laboratory", Am. J. Phys., 80, pp. 497-505 (2012). 